\title{
Early Recurrence After Pulmonary Vein Isolation of Paroxysmal Atrial Fibrillation With Different Ablation Technologies - Prospective Comparison of Radiofrequency vs. Second-Generation Cryoballoon Ablation -
}

\author{
Shinsuke Miyazaki, MD; Akio Kuroi, MD; Hitoshi Hachiya, MD; \\ Hiroaki Nakamura, MD; Hiroshi Taniguchi, MD; Noboru Ichihara, MD; \\ Takamitsu Takagi, MD; Jin Iwasawa, MD; Yoshito Iesaka, MD
}

\begin{abstract}
Background: Inflammation plays a prominent role in the etiology of the early recurrence of atrial fibrillation (ERAF). We prospectively compared the proportion of ERAF and time-course patterns of biomarkers between radiofrequency (RF) and cryoballoon (CB) ablation.
\end{abstract}

\begin{abstract}
Methods and Results: We enrolled 82 consecutive paroxysmal AF patients undergoing pulmonary vein (PV) isolation, performed with either a 28-mm 2nd-generation CB and 3-min freeze technique or point-by-point RF ablation. Each group had 41 patients. In the RF group, all PVs were successfully isolated with $28.9 \pm 6.5$ min of RF delivery. In the CB group, a mean of 5.3 \pm 1.4 applications/patient was delivered. The proportion of ERAF was similar between the groups. The time-course patterns significantly differed between the groups for high-sensitivity $\mathrm{C}$-reactive protein (hs-CRP) value $(\mathrm{P}=0.006)$ and myocardial injury markers $(\mathrm{P}<0.0001)$. Greater myocardial injury was observed in the $\mathrm{CB}$ than in the RF group $(\mathrm{P}<0.0001)$, whereas the peak hs-CRP value was comparable between the groups. The 2-day post-procedure hs-CRP value was the sole factor correlating with ERAF as identified by the multivariable analysis (hazard ratio 1.697; 95\% confidence interval, 1.005-2.865; $\mathrm{P}=0.048$ ) in the $\mathrm{RF}$, but not the CB group.
\end{abstract}

Conclusions: The proportion of ERAF was comparable after RF and 2nd-generation CB ablation. Despite CB ablation exhibiting greater myocardial injury than RF ablation, the inflammatory responses were comparable between the groups. The inflammatory response extent predicted ERAF post-RF ablation but not post-CB ablation. (Circ J 2016; 80: 346-353)

Key Words: Atrial fibrillation; Catheter ablation; Cryoballoon; Early recurrence; Pulmonary vein isolation

C atheter ablation of atrial fibrillation (AF) with the primary aim of pulmonary vein isolation (PVI) is now an effective and safe therapeutic option for patients with paroxysmal AF. ${ }^{1}$ Recently, cryoballoon (CB) ablation has emerged as an alternative technique for PVI, and has gained increasing acceptance as an effective ablation tool. ${ }^{2}$ A multicenter prospective trial (STOP-AF trial) has shown that early recurrence of $\mathrm{AF}$ (ERAF), which is defined as recurrence within a 3-month blanking period post $\mathrm{AF}$ ablation, is commonly observed after 1 st-generation CB ablation, ${ }^{3}$ as with post radiofrequency $(\mathrm{RF})$ ablation. However, to date, there have been no data directly comparing ERAF post-RF ablation and that post-2nd-generation $\mathrm{CB}$ ablation. Although the mechanisms of ERAF remain to be fully elucidated, the results of published data suggest that inflammation plays a prominent role in its etiology. ${ }^{4-6}$ The aim of the present study was to prospectively compare (1) the proportion of patients with AF recurrence within the first 3 months post ablation procedure (ie, ERAF) using a 14-day consecutive monitoring system, (2) the timecourse patterns of biomarkers of myocardial injury, and inflammatory and prothrombotic biomarkers during the perioperative period, and (3) the factors associated with ERAF, in RF and 2nd-generation $\mathrm{CB}$ ablation patients with paroxysmal AF.

\section{Methods}

\section{Study Population}

This prospective study consisted of 82 consecutive patients

Received September 30, 2015; revised manuscript received October 31, 2015; accepted November 5, 2015; released online December 4, 2015 Time for primary review: 26 days

Cardiology Division, Cardiovascular Center, Tsuchiura Kyodo Hospital, Tsuchiura, Japan

Mailing address: Shinsuke Miyazaki, MD, Cardiology Division, Cardiovascular Center, Tsuchiura Kyodo Hospital, 11-7 Manabeshinmachi, Tsuchiura 300-0053, Japan. E-mail: mshinsuke@k3.dion.ne.jp

ISSN-1346-9843 doi:10.1253/circj.CJ-15-1051

All rights are reserved to the Japanese Circulation Society. For permissions, please e-mail: cj@j-circ.or.jp 
with paroxysmal AF who underwent their first PVI between July 2014 and December 2014. The strategy of PVI (either point-by-point RF ablation or 2nd-generation CB ablation) was chosen according to the patients' preference after being informed about both strategies by the physician. AF was classified according to the latest guidelines. ${ }^{7}$ All patients gave written informed consent. The study protocol was approved by the hospital's institutional review board and the study complied with the Declaration of Helsinki.

\section{Mapping and Ablation Protocol}

All antiarrhythmic drugs were discontinued for at least 5 halflives prior to the procedure. Warfarin and novel oral anticoagulants were stopped 1 day before the procedure. The cardiac anatomy was evaluated pre-procedurally using 320-row contrast-enhanced computed tomography. The surface ECG and bipolar intracardiac electrograms were continuously monitored and stored on a computer-based digital recording system (LabSystem PRO, Bard Electrophysiology, Lowell, MA, USA). The bipolar electrograms were filtered from 30 to $500 \mathrm{~Hz}$. A 7Fr 20-pole 3-site mapping catheter (BeeAT, Japan-Life-Line, Tokyo, Japan) was inserted through the right jugular vein for pacing, recording, and internal cardioversion. The procedure was performed under minimal or moderate sedation. ${ }^{8}$ A bolus of $100 \mathrm{IU} / \mathrm{kg}$ body weight of heparin was administered immediately following venous access, and heparinized saline was additionally infused to maintain the activated clotting time at 250-350s. A single transseptal puncture was performed using a RF needle (Baylis Medical, Inc, Montreal, QC, Canada) and 8Fr long sheath (SL0, AF Division, SJM, Minneapolis, MN, USA).

\section{RF Group}

After a transseptal puncture, 2 long sheaths were introduced into both superior PVs. Following pulmonary venography during ventricular pacing, 2 circular mapping catheters (Lasso, Biosense-Webster, Diamond Bar, CA, USA) were placed in the ipsilateral PVs, and the left- and right-sided ipsilateral PVs were circumferentially ablated guided by a 3-D mapping system (CARTO3, Biosense-Webster). ${ }^{9-11}$ Posteriorly, ablation was performed anatomically in the left atrium (LA), approximately $1-3 \mathrm{~cm}$ from the PV ostia. Anteriorly, ablation was performed on the edge of the left PVs guided by the earliest PV potential. The endpoint of the PVI was achieving bidirectional conduction block between the LA and PVs without adenosine-provoked dormant conduction. RF current was delivered point-by-point with a 3.5-mm externally irrigatedtip ablation catheter (Thermocool, Biosense-Webster) with a power of up to $35 \mathrm{~W}$, target temperature of $\leq 38^{\circ} \mathrm{C}$ and irrigation rate of $30 \mathrm{ml} / \mathrm{min}$. The power was limited to $20 \mathrm{~W}$ on the posterior wall close to the esophagus.

\section{Cryoablation Group}

The transseptal sheath was exchanged over a guidewire for a 15Fr steerable sheath (Flexcath Advance, Medtronic, Minneapolis, MN, USA). A spiral mapping catheter (Achieve, Medtronic) was used to advance the 2nd-generation 28-mm CB (Arctic Front Advance, Medtronic) into the PV for support and to map the PV potentials. No 23-mm CBs were used in any cases. Contrast medium was used to verify the complete occlusion of the PV ostium, which was followed by a freeze cycle of $180 \mathrm{~s}$. No additional applications were performed after the isolation. In order to avoid bilateral phrenic nerve injury, ${ }^{12}$ all CB applications were applied under monitoring of ipsilateral diaphragmatic compound motor action potentials during phrenic nerve pacing. The procedural endpoint was defined as electrical PVI verified by the 20 -mm circular mapping catheter (Lasso) without adenosine-provoked dormant conduction. ${ }^{13}$ Additional touch-up freezes with an 8-mm tip conventional cryocatheter (Freezor MAX, Medtronic) were performed with 2 min of each application.

\section{Body Temperature and Biomarkers}

The patients' body temperature was measured every $6 \mathrm{~h}$ during the first 2 consecutive days after the PVI. Blood samples were taken for the total white cell count (WBC), neutrophil/lymphocyte ratio (NLR), high-sensitivity C-reactive protein (hs$\mathrm{CRP})$, troponin-I, creatine kinase-MB (CK-MB), fibrinogen, and D-dimer measurements at 6 different time points: before the procedure, after isolation of the one-sided ipsilateral PVs, after isolation of all PVs, and at 1 and 2 days, and 3 weeks after the procedure.

\section{Follow-up}

No antiarrhythmic drugs were prescribed after the procedure except for patients with highly symptomatic ERAF. The patients underwent continuous, in-hospital ECG monitoring for 2 days following the procedure. Subsequently, 14-day consecutive monitoring using an external loop recorder (Spider Flash, Sorin, France $)^{14}$ was undertaken following discharge. The first outpatient clinic visit was 3 weeks after the ablation procedure. Subsequent follow-up visits consisted of a clinical interview, ECGs, and 24-h Holter monitoring every 1-2 months at the cardiology clinic. Anticoagulation therapy was continued for a minimum of 3 months. Patients with palpitations were encouraged to use a patient-activated event recorder for 1 month. Recurrence was defined according to the patient's symptoms, and/or if an arrhythmia lasting longer than $30 \mathrm{~s}$ was documented, following the latest guidelines.

\section{Statistical Analysis}

Continuous data are expressed as the mean \pm standard deviation for normally distributed variables or as the median [25th, 75 th percentiles] for non-normally distributed variables, and were compared using Student's t-test or Mann-Whitney U-test, respectively. Categorical variables were compared using the chi-square test. The Pearson's correlation coefficient was measured to evaluate the correlation between 2 variables. Parameters with a significance $<0.2$ in the univariate analysis were entered into a multiple logistic regression analysis to identify the factors associated with ERAF. Kaplan-Meier analysis was used to determine the percentage of patients free from recurrence. The difference in the arrhythmia-free survival was evaluated using the log-rank test. The time-course patterns of each parameter were compared with a 2-way layout ANOVA between the RF and CB ablation groups. Sequential data measurements in each group were analyzed by a repeated-measures analysis of variance (repeated-measures ANOVA). A probability value $<0.05$ indicated statistical significance.

\section{Results}

\section{Patient Characteristics and Procedural Results}

Of the 82 patients, $41(50.0 \%)$ underwent RF ablation, and the other $41(50.0 \%)$ underwent CB ablation. There was no significant difference in terms of patient characteristics between the 2 groups (Table 1). In the RF group, all PVs were successfully isolated with a total RF delivery time of $28.9 \pm 6.5 \mathrm{~min}$ and total energy delivery of $47,992 \pm 11,162$ joules. In the CB 


\begin{tabular}{lccc}
\hline \multicolumn{2}{l}{ Table 1. Characteristics of the Study Population of Patients Undergoing AF Ablation } & \\
$\mathrm{n}$ & RF group & CB group & P value \\
Age, years & 41 & 41 & \\
Female, $\mathrm{n}(\%)$ & $63.7 \pm 10.8$ & $61.4 \pm 10.5$ & 0.322 \\
Structural heart disease, $\mathrm{n}(\%)$ & $13(31.7)$ & $11(26.8)$ & 0.627 \\
Hypertension, $\mathrm{n}(\%)$ & $2(4.9)$ & $4(9.7)$ & 0.396 \\
$\mathrm{BMl}, \mathrm{kg} / \mathrm{m}^{2}$ & $19(46.3)$ & $23(56.1)$ & 0.377 \\
$\mathrm{CHADS}$ score & $23.8 \pm 3.1$ & $24.1 \pm 2.7$ & 0.593 \\
CHA2DS2-VASc score & $0.88 \pm 0.92$ & $1.07 \pm 1.12$ & 0.394 \\
LA diameter, mm & $1.61 \pm 1.24$ & $1.71 \pm 1.38$ & 0.738 \\
LVEF, \% & $37.8 \pm 4.3$ & $38.7 \pm 4.6$ & 0.345 \\
Pro-BNP, pg/ml & $65.7 \pm 7.6$ & $65.7 \pm 7.7$ & 0.974 \\
eGFR, ml/min/1.73 m ${ }^{2}$ & $164 \pm 289$ & $192 \pm 328$ & 0.699 \\
\hline
\end{tabular}

$A F$, atrial fibrillation; BMI, body mass index; BNP, B-type natriuretic peptide; $C B$, cryoballoon; eGFR, estimated glomerular filtration rate; LA, left atrial; LVEF, left ventricular ejection fraction; RF, radiofrequency.

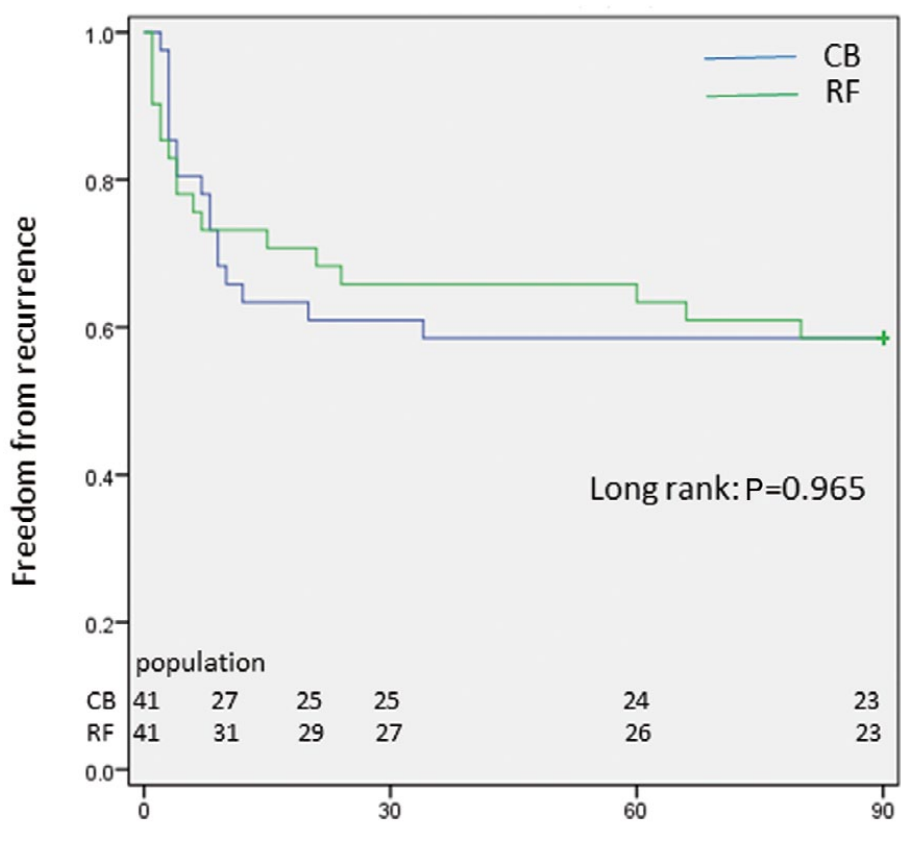

Time to ERAF from index ablation procedure (days)
Figure 1. Time to the first recurrence of atrial fibrillation (AF) within the first 3 months of the index radiofrequency (RF) or cryoballoon (CB) ablation. ERAF, early recurrence of AF. group, 156 of 167 (93.4\%) PVs were isolated successfully using exclusively $28-\mathrm{mm}$ 2nd-generation CBs. The total number of CB applications was 294 (mean 5.3 $\pm 1.4 /$ patient), and the mean number of $\mathrm{CB}$ applications resulting in PVI was $1.1 \pm 0.4,1.0,1.3 \pm 0.5,1.1 \pm 0.5$, and $1.2 \pm 0.5$ for the right superior, right middle, right inferior, left superior, and left inferior $\mathrm{PV}$, respectively. Touch-up focal lesions were created in the remaining $11(6.6 \%) \mathrm{PVs}$, and the median number of applications to achieve PVI was 3 (2-4). Right phrenic nerve injury, and a pneumothorax during the puncture of the right subclavian vein occurred in 1 patient in the $\mathrm{CB}$ group.

\section{Incidence of ERAF}

The freedom from recurrent atrial tachyarrhythmias within 3 months post-procedure is shown in Figure 1. There was no significant difference in freedom from ERAF between the RF and $\mathrm{CB}$ groups $(\mathrm{P}=0.965)$. Among the 34 patients with ERAF, it was detected during the initial 16-day continuous monitoring period post-procedure in $27(79.4 \%)$. Time to the initial ERAF was a median of 5.0 (3.0-12.8) days, and was asymptomatic in $15(44.1 \%)$ patients. There was no significant difference between the RF and $\mathrm{CB}$ groups in the time to the initial $\operatorname{ERAF}(\mathrm{P}=0.169)$ and prevalence of asymptomatic recurrences $(\mathrm{P}=0.373)$. The patients with asymptomatic ERAF were more likely to have hypertension and lower renal function than the patients with symptomatic ERAF (Table 2).

\section{Time-Course of Body Temperature and Biomarkers}

The inflammation, myocardial injury, and prothrombotic marker time courses are shown in Figures 2-4. The baseline values 
Table 2. Characteristics of the Patients With Symptomatic and Asymptomatic ERAF

n

Age, years

Female, $\mathrm{n}(\%)$

Structural heart disease, $\mathrm{n}(\%)$

Hypertension, $\mathrm{n}(\%)$

$\mathrm{BMl}, \mathrm{kg} / \mathrm{m}^{2}$

$\mathrm{CHADS}_{2}$ score

$\mathrm{CHA}_{2} \mathrm{DS}_{2}$-VASc score

LA diameter, $\mathrm{mm}$

LVEF, \%

Pro-BNP, $\mathrm{pg} / \mathrm{ml}$

eGFR, $\mathrm{ml} / \mathrm{min} / 1.73 \mathrm{~m}^{2}$

\section{Symptomatic}

19

$63.3 \pm 11.0$

$6(31.6)$

2 (10.5)

6 (31.6)

$24.0 \pm 1.7$

$0.73 \pm 0.99$

$1.52 \pm 1.22$

$38.4 \pm 4.7$

$65.6 \pm 5.5$

$162 \pm 296$

$77.3 \pm 18.8$
Asymptomatic

15

$62.1 \pm 6.6$

$6(40.0)$

$3(20.0)$

$10(66.7)$

$24.6 \pm 3.6$

$1.40 \pm 1.05$

$1.86 \pm 1.12$

$39.6 \pm 5.0$

$63.4 \pm 10.3$

$274 \pm 401$

$62.5 \pm 10.4$
P value

0.716

0.610

0.438

0.042

0.523

0.069

0.409

0.457

0.451

0.369

0.010

ERAF, early recurrence of atrial fibrillation. Other abbreviations as in Table 1.
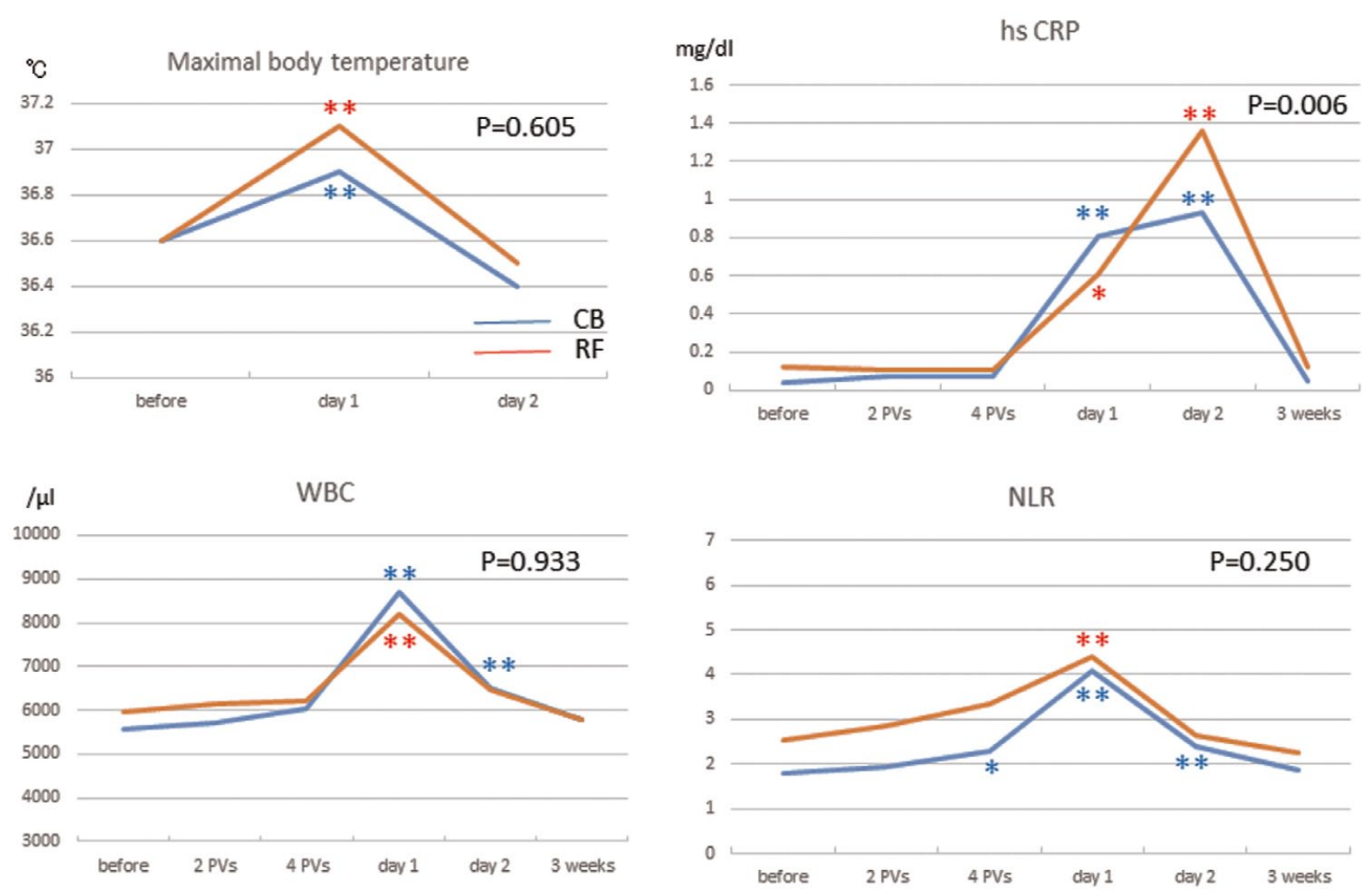

Figure 2. Time course of body temperature and inflammatory markers after radiofrequency (RF) or cryoballoon (CB) ablation. ${ }^{\star} \mathrm{P}<0.05,{ }^{\star}{ }^{*} \mathrm{P}<0.001$ (compared with baseline), $\mathrm{P}$ values indicate the comparison of the time-course pattern between the RF and $\mathrm{CB}$ ablation groups. hs-CRP, high-sensitivity C-reactive protein; NLR, neutrophil/lymphocyte ratio; WBC, total white cell count.

of each parameter were similar between the 2 groups (Table 3 ). Body temperature, total WBC, NLR, CK-MB, troponin-I, and D-dimer levels peaked 1 day post-procedure in both groups. The hs-CRP and fibrinogen levels peaked 2 days post-procedure in both groups. The time-course patterns significantly differed between the 2 groups for CK-MB $(\mathrm{P}<0.0001)$, troponin-I $(\mathrm{P}<0.0001)$, and hs-CRP levels $(\mathrm{P}=0.006)$, but not the other markers. There was no significant difference between the 2 groups for the hs-CRP value 1 day $(\mathrm{P}=0.256)$, and 2 days $(\mathrm{P}=0.127)$ post-procedure. In the $\mathrm{RF}$ group, the amount of total RF energy delivered did not correlate with the hs-CRP value either 1 day $\left(\mathrm{R}^{2}=0.056, \mathrm{P}=0.144\right)$ or 2 days $\left(\mathrm{R}^{2}=0.000\right.$, $\mathrm{P}=0.914)$ post-procedure.

\section{Factors Associated With ERAF}

The hs-CRP value 2 days post-procedure was the sole factor 

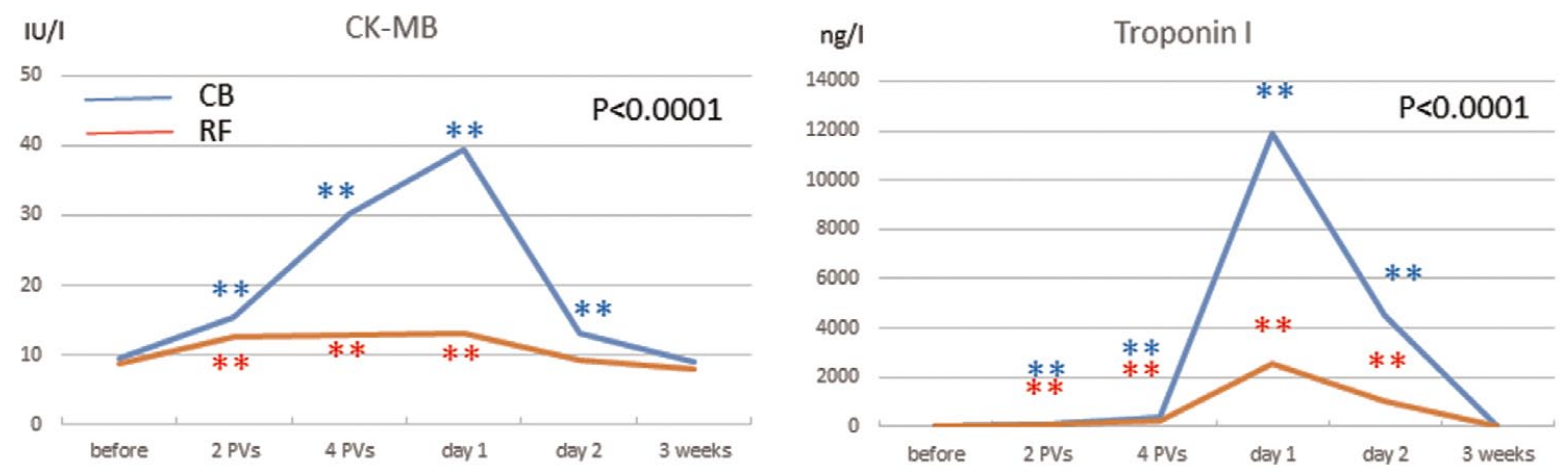

Figure 3. Time course of the markers of myocardial injury after radiofrequency (RF) or cryoballoon (CB) ablation. ${ }^{* *} \mathrm{P}<0.001$ (compared with baseline), $\mathrm{P}$ values indicate the comparison of the time-course pattern between the RF and CB ablation group.

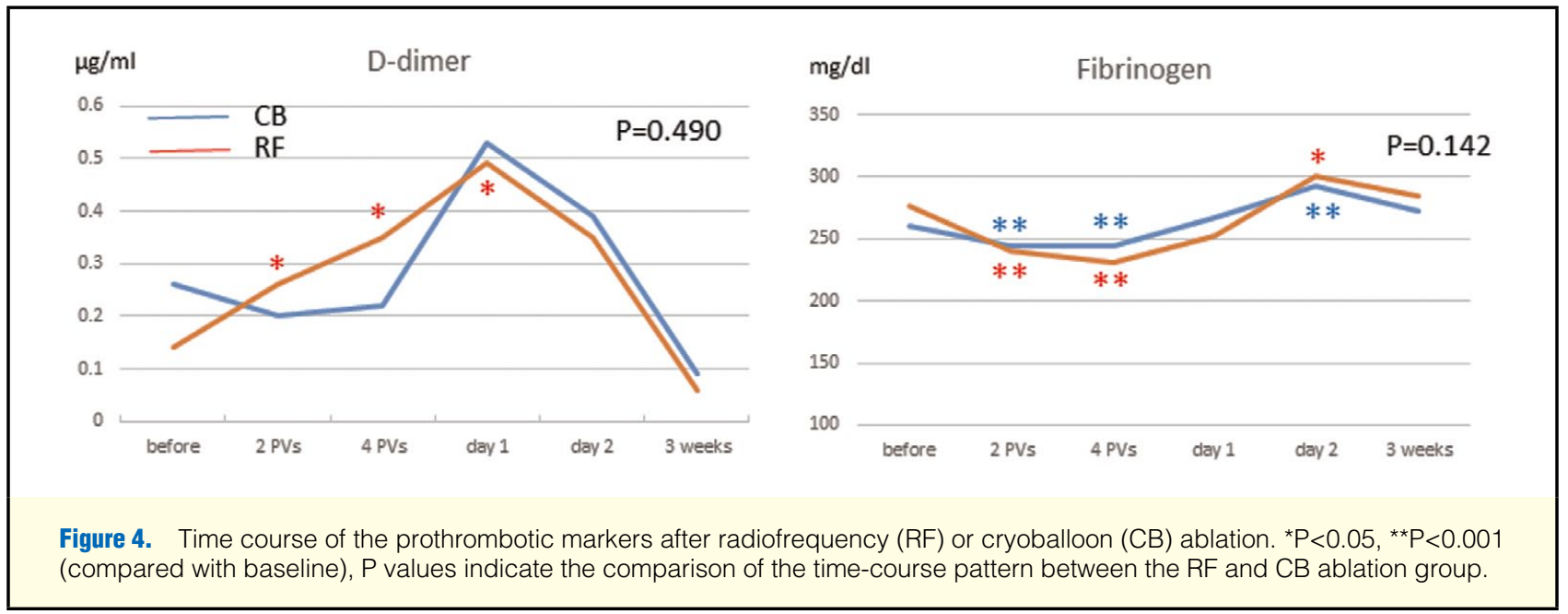

\begin{tabular}{|lccc|}
\hline \multicolumn{4}{|c|}{ Table 3. Baseline Value of Each Biomarker in the RF and CB Groups of Patients Undergoing AF Ablation } \\
& RF group & CB group & P value \\
WBC, $/ \mu \mathrm{l}$ & $5,959 \pm 1,683$ & $5,562 \pm 1,260$ & 0.232 \\
$\mathrm{hs}-\mathrm{CRP}, \mathrm{mg} / \mathrm{dl}$ & $0.12 \pm 0.30$ & $0.04 \pm 0.06$ & 0.076 \\
$\mathrm{NLR}$ & $2.54 \pm 2.48$ & $1.81 \pm 0.64$ & 0.070 \\
$\mathrm{CK}-\mathrm{MB}, \mathrm{IU} / \mathrm{L}$ & $8.8 \pm 2.5$ & $9.5 \pm 3.0$ & 0.273 \\
Troponin-l, $\mathrm{ng} / \mathrm{L}$ & $3.7 \pm 4.9$ & $4.3 \pm 11$ & 0.737 \\
D-dimer, $\mu \mathrm{g} / \mathrm{ml}$ & $0.14 \pm 0.32$ & $0.26 \pm 0.9$ & 0.436 \\
Fibrinogen, $\mathrm{mg} / \mathrm{dl}$ & $277 \pm 55$ & $260 \pm 46$ & 0.133 \\
\hline
\end{tabular}

hs-CRP, high-sensitivity C-reactive protein; WBC, white blood cell count. Other abbreviations as in Table 1.

correlating with ERAF as identified by univariate (hazard ratio (HR) $1.474 ; 95 \%$ confidence interval (CI), 1.020-2.128; $\mathrm{P}=0.039)$ and multivariable analyses (HR $1.578 ; 95 \% \mathrm{CI}$, $1.071-2.325 ; \mathrm{P}=0.021)$ in all patients. Similarly, the hs-CRP value 2 days post-procedure was the sole factor correlating with ERAF as identified by the multivariable analysis (HR 1.697 ; 95\% CI, 1.005-2.865; P=0.048; Tables 4,S1) in the RF group. However, no factors, including the hs-CRP, correlated with ERAF in the CB group.

\section{Clinical Outcome}

When a 3-month blanking period was applied, the freedom from any recurrent atrial arrhythmias off antiarrhythmic drugs after a single procedure was significantly higher in the $\mathrm{CB}$ 


\begin{tabular}{|c|c|c|c|c|c|c|}
\hline & \multicolumn{3}{|c|}{ Univariate analysis } & \multicolumn{3}{|c|}{ Multivariate analysis } \\
\hline & HR & $95 \% \mathrm{Cl}$ & $P$ value & HR & $95 \% \mathrm{Cl}$ & $P$ value \\
\hline \multicolumn{7}{|l|}{ All patients } \\
\hline LA diameter, mm & & & 0.174 & & & 0.376 \\
\hline LVEF, \% & & & 0.119 & & & 0.101 \\
\hline CRP day 2 & 1.474 & $1.020-2.128$ & 0.039 & 1.578 & $1.071-2.325$ & 0.021 \\
\hline \multicolumn{7}{|l|}{ RF group } \\
\hline LA diameter, mm & & & 0.124 & & & 0.198 \\
\hline CRP day 2 & & & 0.072 & 1.697 & $1.005-2.865$ & 0.048 \\
\hline \multicolumn{7}{|l|}{ CB group } \\
\hline Sex & & & 0.165 & & & 0.220 \\
\hline Structural heart disease & & & 0.185 & & & 0.235 \\
\hline
\end{tabular}

$\mathrm{Cl}$, confidence interval; $\mathrm{HR}$, hazard ratio. Other abbreviations as in Tables 1-3.

than in the RF group (37/41 vs. $28 / 41, \mathrm{P}=0.014)$ during $11.0 \pm 3.2$ months of follow-up. In the $\mathrm{CB}$ group, the success rate was significantly higher in the patients without ERAF than in those with it $(24 / 24$ vs. $13 / 17, \mathrm{P}=0.012)$. In the $\mathrm{RF}$ group, the success rate was also significantly higher in the patients without ERAF than in those with it $(20 / 24$ vs. $8 / 17, \mathrm{P}=0.014)$.

\section{Discussion}

To the best of our knowledge, this is the first study to prospectively compare the proportion of ERAF and time-course of biomarkers during the perioperative period of PVI using RF and 2nd-generation CBs. We found that (1) the proportion of ERAF was similar between the groups, (2) approximately half of the ERAF was asymptomatic, and the times to the initial ERAF and prevalence of asymptomatic recurrences were similar between the groups, (3) myocardial injury was significantly greater for CB than RF ablation, (4) the time-course pattern of the hs-CRP value differed between the groups, and (5) the hs-CRP value post PVI was the sole factor associated with ERAF for RF ablation, but not $\mathrm{CB}$ ablation.

\section{Markers of Myocardial Injury and Prothrombotic Markers}

The myofibrillar protein, troponin-I, is a sensitive and specific marker of myocyte injury, and an elevated level reflects the amount of myocardial destruction. Higher troponin levels post PVI were reported when using the 1st-generation CB instead of RF catheters, ${ }^{15}$ and with the 2 nd-generation instead of the 1st-generation $\mathrm{CB} .{ }^{16}$ Creating complete occlusion of the PV may result in minimized convective warming of the local blood velocity and may create more extended lesions, as indicated by the higher troponin-I level. Moreover, the recently introduced 2nd-generation CBs provide more uniform and distal cooling than the 1st-generation balloons because of improved cooling of the distal balloon hemisphere. ${ }^{17}$ Our finding that the 2nd-generation CB resulted in a more pronounced myocardial injury than RF ablation despite the short application time was in line with the previous reported results.

On the other hand, the measured levels and patterns of change of the prothrombotic markers were comparable between the present study groups. Malmborg et al did not find any major differences in the overall activation of the coagulation system between cryoablation (1st-generation $\mathrm{CB}$ ) and RF ablation (multielectrode RF catheter), even though the CB caused more pronounced myocardial destruction than RF. ${ }^{18}$ Our result of comparable prothrombotic marker values despite more pro- nounced myocardial destruction with 2nd-generation CB ablation, was in accordance with that finding.

\section{Markers of Inflammation}

Elevation of the WBC, NLR, hs-CRP, and body temperature values suggests a systemic inflammatory process in addition to local inflammation after catheter ablation. NLR provides information on the inflammatory status and has been studied in several clinical observational studies of cardiovascular diseases. ${ }^{19}$ In the present study, inflammatory responses were similarly observed in both groups; however, the hs-CRP timecourse pattern differed between them. RF ablation produces thermal necrosis, resulting in localized coagulative necrosis of the atrial myocardium. Inflammatory reactions around the injury sites, with infiltration of inflammatory cells, have been found in histopathologic studies. ${ }^{20-22}$ In the RF group, the hsCRP value post-procedure did not correlate with the total amount of RF energy delivered, presumably because the inflammatory reaction differs among individual patients and the amount of tissue injury is defined by several parameters, such as the contact force. Cryothermal tissue injury was found to be distinguished from hyperthermic injury by less inflammation through preservation of the basic underlying tissue architecture and minimal thrombus formation. ${ }^{21,23,24}$ Cryotherapy was shown to induce irreversible injury with transparietal degenerative nucleocytoplasmic cell changes. Our study showed that the hs-CRP level was comparable between the 2 groups despite higher levels of myocardial injury in the $\mathrm{CB}$ group, which is in accordance with the results after cavotricuspid isthmus ablation. ${ }^{25}$ Relatively lower hs-CRP levels might be related to the lack of endothelial damage with cryoablation, whereas the freeze/thaw process with cardiomyocyte swelling and membrane rupture might induce greater release of myocardial injury markers. Prior studies speculated that the inflammatory response could be partly explained by local myocardial injury in RF ablation because of the positive correlation between hs-CRP elevation and markers of myocardial injury, ${ }^{6}$ but according to our study results that did not seem applicable to $\mathrm{CB}$ ablation.

\section{ERAF After RF and CB Ablation}

The mechanisms of ERAF remain to be fully elucidated. Richter et al demonstrated that markers of oxidative stress linked to inflammation showed significant upregulation during the first 2 days after AF ablation and predicted a recurrence within 1 week post-ablation, but not the long-term outcome. ${ }^{5}$ 
Koyama et al demonstrated that corticosteroid therapy during the initial 3 days post-ablation significantly decreased the inflammation and recurrent arrhythmias within 3 days postablation. ${ }^{4}$ Lim et al reported that an elevation of inflammatory markers predicted recurrent arrhythmias within 3 days of the procedure, but not at 3 and 6 months. ${ }^{6}$ Those findings support the hypothesis that inflammation plays a prominent role in the etiology of ERAF after RF ablation.

From this standpoint, the incidence and significance of ERAF may differ between RF and CB ablation. ${ }^{26}$ Acute thermal injury effected by RF is characterized by coagulation and tissue necrosis and is followed by a marked inflammatory response, which is thought to be mechanistically central to the occurrence of ERAF. In contrast, cryoablation results in the creation of well-demarcated homogeneous lesions through a directed freezing process. ${ }^{23}$ As a result, it can be postulated that cryothermal ablation should result in a lower rate of ERAF because of the relatively reduced inflammatory reaction. Nevertheless, the results of the STOP AF trial revealed that ERAF was a common finding even after 1st-generation $\mathrm{CB}$ ablation. ${ }^{3}$ Our study is the first study to show the incidence of ERAF after 2nd-generation CB ablation, and clarified that the proportion of ERAF cases was similar for RF and 2ndgeneration $\mathrm{CB}$ ablation. In line with the prior studies, the recurrent episodes were commonly asymptomatic, and the time to the first ERAF was within 2 weeks post-procedure in the majority of cases. The result emphasizes the importance of rigorous monitoring post-ablation regardless of the ablation technology used.

The present data also demonstrated a positive correlation between hs-CRP elevation and ERAF for RF ablation, which is compatible with prior study results. ${ }^{8-10}$ In contrast, no factors that correlated with ERAF could be identified for $\mathrm{CB}$ ablation. The finding that the time-course of hs-CRP levels differed between the groups and that the inflammatory markers did not correlate with ERAF for $\mathrm{CB}$ ablation suggested that the mechanism of ERAF cannot be simply explained by inflammatory process alone. It is also possible that hs-CRP is not sufficiently sensitive for evaluating the inflammatory status after CB ablation. The STOP-AF study showed that (1) ERAF after $\mathrm{CB}$ ablation significantly correlated with late recurrence, (2) a significant proportion of patients undergoing repeat ablation had early reconnections (52\% of PVs), and (3) an early repeat ablation was associated with excellent longterm freedom from recurrent AF. ${ }^{3}$ Although our study population was small and the follow-up period was not long, ERAF correlated with late recurrences in both groups, which was in line with the prior studies. ${ }^{3,10}$ Although the exact mechanism of ERAF post-CB ablation is unknown, it is likely that the inflammatory response, $\mathrm{PV}$ reconnections, non-PV foci, and unknown factors are associated with ERAF after CB ablation. A more sensitive marker reflecting the inflammatory status might be required for further evaluation. Further studies should be conducted to clarify the mechanism of ERAF in CB ablation.

\section{Study Limitations}

The study was a single-center non-randomized study and the population was relatively small. Therefore, the study might have a potential selection bias. The different freezing times could have affected the study results; however, recent clini$\mathrm{cal}^{27}$ and experimental ${ }^{28}$ studies showed that single 3-min freeze techniques, without extra applications, are effective in achieving acute PVI and AF freedom $>80 \%$ at 1-year followup when using 2nd-generation CB.

\section{Conclusion}

The proportion of ERAF cases after paroxysmal AF ablation was comparable for RF and 2nd-generation CB ablation. Despite $\mathrm{CB}$ ablation exhibiting greater myocardial injury than RF ablation, the inflammatory response was comparable between the groups. The extent of the inflammatory response predicted ERAF post-RF ablation but not post-CB ablation.

\section{Acknowledgments}

We thank Mr John Martin for his help in the preparation of the manuscript.

\section{Conflicts of Interest / Financial Support}

None.

\section{References}

1. Haïssaguerre M, Jaïs P, Shah DC, Garrigue S, Takahashi A, Lavergne $\mathrm{T}$, et al. Electrophysiological end point for catheter ablation of atrial fibrillation initiated from multiple pulmonary venous foci. Circulation 2000; 101: 1409-1417.

2. Kojodjojo P, O'Neill MD, Lim PB, Malcolm-Lawes L, Whinnett ZI, Salukhe TV, et al. Pulmonary venous isolation by antral ablation with a large cryoballoon for treatment of paroxysmal and persistent atrial fibrillation: Medium-term outcomes and non-randomised comparison with pulmonary venous isolation by radiofrequency ablation. Heart 2010; 96: $1379-1384$.

3. Andrade JG, Khairy P, Macle L, Packer DL, Lehmann JW, Holcomb $\mathrm{RG}$, et al. Incidence and significance of early recurrences of atrial fibrillation after cryoballoon ablation: Insights from the multicenter Sustained Treatment of Paroxysmal Atrial Fibrillation (STOP AF) Trial. Circ Arrhythm Electrophysiol 2014; 7: 69-75.

4. Koyama T, Tada H, Sekiguchi Y, Arimoto T, Yamasaki H, Kuroki $\mathrm{K}$, et al. Prevention of atrial fibrillation recurrence with corticosteroids after radiofrequency catheter ablation: A randomized controlled trial. J Am Coll Cardiol 2010; 56: 1463-1472.

5. Richter B, Gwechenberger M, Socas A, Zorn G, Albinni S, Marx M, et al. Markers of oxidative stress after ablation of atrial fibrillation are associated with inflammation, delivered radiofrequency energy and early recurrence of atrial fibrillation. Clin Res Cardiol 2012; 101: $217-225$.

6. Lim HS, Schultz C, Dang J, Alasady M, Lau DH, Brooks AG, et al. Time course of inflammation, myocardial injury, and prothrombotic response after radiofrequency catheter ablation for atrial fibrillation. Circ Arrhythm Electrophysiol 2014; 7: 83-89.

7. Calkins H, Kuck KH, Cappato R, Brugada J, Camm AJ, Chen SA, et al. 2012 HRS/EHRA/ECAS expert consensus statement on catheter and surgical ablation of atrial fibrillation: Recommendations for patient selection, procedural techniques, patient management and follow-up, definitions, endpoints, and research trial design: A report of the Heart Rhythm Society (HRS) Task Force on Catheter and Surgical Ablation of Atrial Fibrillation. Heart Rhythm 2012; 9: 632-696.

8. Ichihara N, Miyazaki S, Taniguchi H, Usui E, Takagi T, Iwasawa J, et al. Simple minimal sedation for catheter ablation of atrial fibrillation. Circ J 2015; 79: 346-350.

9. Miyazaki S, Kuwahara T, Kobori A, Takahashi Y, Takei A, Sato A, et al. Long-term clinical outcome of extensive pulmonary vein isolation-based catheter ablation therapy in patients with paroxysmal and persistent atrial fibrillation. Heart 2011; 97: 668-673.

10. Miyazaki S, Taniguchi H, Nakamura H, Takagi T, Iwasawa J, Hachiya $\mathrm{H}$, et al. Clinical significance of early recurrence after pulmonary vein antrum isolation in paroxysmal atrial fibrillation: Insight into the mechanism. Circ J 2015; 79: 2353-2359.

11. Miyazaki S, Taniguchi H, Kusa S, Uchiyama T, Nakamura H, Hachiya $\mathrm{H}$, et al. Impact of atrial fibrillation termination site and termination mode in catheter ablation on arrhythmia recurrence. Circ J 2014; 78: 78-84.

12. Miyazaki S, Hachiya $H$, Taniguchi $H$, Nakamura $H$, Ichihara N, Usui E, et al. Prospective evaluation of bilateral diaphragmatic electromyograms during cryoballoon ablation of atrial fibrillation. $J$ Cardiovasc Electrophysiol 2015; 26: 622-628.

13. Miyazaki S, Taniguchi $\mathrm{H}$, Nakamura $\mathrm{H}$, Hachiya $\mathrm{H}$, Ichihara $\mathrm{N}$, Araki M, et al. Adenosine triphosphate test after cryothermal pulmonary vein isolation: Creating contiguous lesions is essential for 
eliminating dormant conduction. J Cardiovasc Electrophysiol 2015; 26: $1069-1074$.

14. Takagi T, Miyazaki S, Kusa S, Taniguchi H, Ichihara N, Iwasawa J, et al. Role of extended external auto-triggered loop recorder monitoring for atrial fibrillation: Validation and utility after catheter ablation procedures. Circ J 2014; 78: 2637-2642.

15. Schmidt M, Marschang H, Clifford S, Harald R, Guido R, Oliver T, et al. Trends in inflammatory biomarkers during atrial fibrillation ablation across different catheter ablation strategies. Int $J$ Cardiol 2012; 158: 33-38.

16. Bordignon S, Fürnkranz A, Dugo D, Perrotta L, Gunawardene M, Bode $\mathrm{F}$, et al. Improved lesion formation using the novel $28 \mathrm{~mm}$ cryoballoon in atrial fibrillation ablation: Analysis of biomarker release. Europace 2014; 16: 987-993.

17. Coulombe N, Paulin J, Su W. Improved in vivo performance of second-generation cryoballoon for pulmonary vein isolation. J Cardiovasc Electrophysiol 2013; 24: 919-925.

18. Malmborg H, Lönnerholm S, Blomström P, Blomström-Lundqvist C. Ablation of atrial fibrillation with cryoballoon or duty-cycled radiofrequency pulmonary vein ablation catheter: A randomized controlled study comparing the clinical outcome and safety; the AFCOR study. Europace 2013; 15: 1567-1573.

19. Bhat T, Teli S, Rijal J, Bhat H, Raza M, Khoueiry G, et al. Neutrophil to lymphocyte ratio and cardiovascular diseases: A review. Expert Rev Cardiovasc Ther 2013; 11: 55-59.

20. Grubman E, Pavri BB, Lyle S, Reynolds C, Denofrio D, Kocovic DZ. Histopathologic effects of radiofrequency catheter ablation in previously infarcted human myocardium. $J$ Cardiovasc Electrophysiol 1999; 10: 336-342.

21. Rodriguez LM, Leunissen J, Hoekstra A, Korteling BJ, Smeets JL, Timmermans C, et al. Transvenous cold mapping and cryoablation of the AV node in dogs: Observations of chronic lesions and comparison to those obtained using radiofrequency ablation. $J$ Cardiovasc Electrophysiol 1998; 9: 1055-1061.

22. Deneke T, Khargi K, Müller KM, Lemke B, Mügge A, Laczkovics A, et al. Histopathology of intraoperatively induced linear radiofrequency ablation lesions in patients with chronic atrial fibrillation.
Eur Heart J 2005; 26: 1797-1803.

23. Khairy P, Chauvet P, Lehmann J, Lambert J, Macle L, Tanguay JF, et al. Lower incidence of thrombus formation with cryoenergy versus radiofrequency catheter ablation. Circulation 2003; 107: 20452050.

24. Dubuc M, Roy D, Thibault B, Ducharme A, Tardif JC, Villemaire C, et al. Transvenous catheter ice mapping and cryoablation of the atrioventricular node in dogs. Pacing Clin Electrophysiol 1999; 22: $1488-1498$.

25. Oswald H, Gardiwal A, Lissel C, Yu H, Klein G. Difference in humoral biomarkers for myocardial injury and inflammation in radiofrequency ablation versus cryoablation. Pacing Clin Electrophysiol 2007; 30: 885-890.

26. Hernández-Romero D, Marín F, Roldán V, Peñafiel P, Vilchez JA, Orenes-Piñero E, et al. Comparative determination and monitoring of biomarkers of necrosis and myocardial remodeling between radiofrequency ablation and cryoablation. Pacing Clin Electrophysiol 2013; 36: 31-36.

27. Ciconte G, de Asmundis C, Sieira J, Conte G, Di Giovanni G, Mugnai G, et al. Single 3-minute freeze for second-generation cryoballoon ablation: One-year follow-up after pulmonary vein isolation. Heart Rhythm 2015; 12: 673-680.

28. Takami M, Lehmann HI, Misiri J, Parker KD, Sarmiento RI, Johnson $\mathrm{SB}$, et al. The impact of freezing time and balloon size on the thermodynamics and isolation efficacy during pulmonary vein isolation using the 2nd generation cryoballoon. Circ Arrhythm Electrophysiol 2015; 8: $836-845$.

\section{Supplementary Files}

Supplementary File 1

Table S1. Factors associated with ERAF after catheter ablation in the univariate analysis ( $\mathrm{P}$ value)

Please find supplementary file(s);

http://dx.doi.org/10.1253/circj.CJ-15-1051 